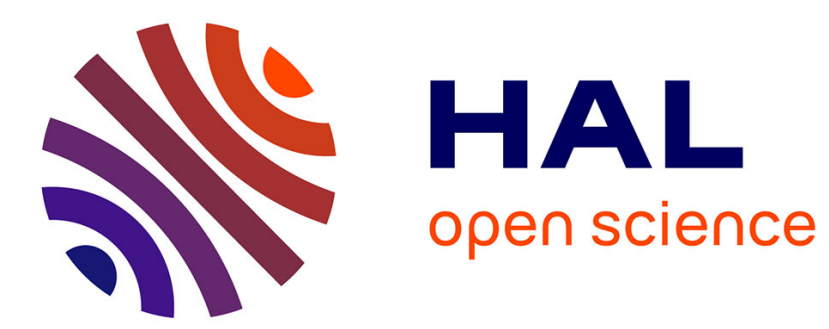

\title{
Quelques remarques sur le rôle de l'outil mathématique dans les études intégrées de paysage
}

Georges Bertrand

\section{To cite this version:}

Georges Bertrand. Quelques remarques sur le rôle de l'outil mathématique dans les études intégrées de paysage. Espace Géographique, 1973, 2 (3), pp.197-198. 10.3406/spgeo.1973.1401 . hal-02570241

\section{HAL Id: hal-02570241 \\ https://hal.science/hal-02570241}

Submitted on 11 May 2020

HAL is a multi-disciplinary open access archive for the deposit and dissemination of scientific research documents, whether they are published or not. The documents may come from teaching and research institutions in France or abroad, or from public or private research centers.
L'archive ouverte pluridisciplinaire HAL, est destinée au dépôt et à la diffusion de documents scientifiques de niveau recherche, publiés ou non, émanant des établissements d'enseignement et de recherche français ou étrangers, des laboratoires publics ou privés. 


\section{Quelques remarques sur le rôle de l'outil mathématique dans les} études intégrées de paysage

\section{Georges Bertrand}

\section{Citer ce document / Cite this document :}

Bertrand Georges. Quelques remarques sur le rôle de l'outil mathématique dans les études intégrées de paysage. In: Espace géographique, tome 2, n³, 1973. pp. 197-198;

doi : https://doi.org/10.3406/spgeo.1973.1401

https://www.persee.fr/doc/spgeo_0046-2497_1973_num_2_3_1401

Fichier pdf généré le 02/04/2018 
R.A. RAPPAPORT, 1971, The flow of energy in an agricultural society. In Energy and power. San Francisco, Freeman.

J.F. RICHARD, 1972, Recherches sur le contact forêt-savane en Côte d'Ivoire. Problèmes de géographie du paysage. In Essai de définition théorique de la géographie $d u$ paysage. Paris, ORSTOM ( $2^{*}$ éd. 1973 pour la R.C.P. n" 231).
M. Stoupy, 1972, Analyse écologique et cartographique du paysage. Contribution méthodologique à l'étude de la distribution spatiale de l'utilisation $d u$ sol. Thèse Doc. Ing., Univ. Paul Sabatier, Toulouse.

G.M. Woodell, 1970, The energy cycle of the biosphere. In The Biosphere. San Francisco, Freeman.

\section{Note critique}

La fin vaut-elle les moyens? G. Allaire, M. Phipps et M. Stoupy d'un côté, J.P. Massonie, D. Mathieu et J.C. Wieber de l'autre, ont imaginé, construit, alimenté et fait fonctionner d'énormes programmes pour traiter numériquement quelques portions restreintes d'espaces agricoles ou «semi-naturels».

Prenons l'exemple de Broze (vallée du Tarn) étudié par la première équipe. Le résultat brut, c'est-à-dire les cartes des classes de sites ou des types de stations, obtenues après l'équivalent de six mois de travail par deux chercheurs à temps plein, et qui avaient à leur disposition tout l'héritage méthodologique de $M$. Phipps, auraient pu être acquis par ces deux mêmes chercheurs, agronomes de formation, en moins d'un mois de terrain et d'enquête auprès des agriculteurs et des services agricoles. On a pu affirmer, très doctement, que «la montagne avait accouché d'une souris». Le problème de l'analyse quantitative appliquée au paysage est ainsi posé. En porteà-faux. Et non sans hypocrisie. Il est vrai qu'on est habitué à juger d'une recherche, même nouvelle, par ses résultats concrets plus que pour sa méthode. Or, dans les deux cas cités plus haut, l'apport méthodologique est d'un intérêt bien supérieur au résultat brut. Il aurait même été souhaitable que les paysages analysés aient été au préalable étudiés par les méthodes «qualitatives» habituelles, pour que soit levée l'hypothèque du «résultat ».

Quel est donc, pour l'instant, et pour un non initié, cet apport méthodologique?

La logique interne du raisonnement et de la démarche analytique. Dans les études intégrées de paysage, on se trouve en présence d'une multiplicité de facteurs (ou d'éléments) dont il est toujours hasardeux de déterminer la hiérarchisation (par exemple, la détermination d'un facteur limitant, d'un correctif écologique, etc.). La question est résolue, le plus souvent correctement - mais on n'en a jamais la certitude -, par l' «intégrateur personnel ». Mais, si cette opération fournit un résultat, elle n'en donne jamais le cheminement. Le raisonnement en souffre, car certains maillons de la chaine logique restent inconscients, donc perdus pour l'analyse. En déterminant les structures d'ordre et en les matérialisant (graphes), l'analyse quantitative livre des classifications et des typologies non seulement contrôlées, mais surtout contrôlables en permanence. Toutefois, «ces hiérarchies sont plus descriptives qu'explicatives »(G. Allaire).

La cohérence par l'homogénéisation de matériel traité. Analyser un paysage, c'est rassembler des données floristiques, pédologiques, humaines et économiques, géomorphologiques, bioclimatiques, juridiques... La procédure numérique (cf. les tableaux de J.C. Wieber) réduit cette hétérogénéité et permet un traitement unique de l'ensemble des données.

Le choix des descripteurs. Ce point pourrait paraître l'un des plus faibles de la méthode. Le choix des variables est compliqué par le passage du «qualitatif 》 au «quantitatif» (plus exactement au numérique). Le problème a au moins le mérite d'être posé dans ce type de recherche, alors qu'il est résolu implicitement dans l'analyse qualitative des milieux naturels et que, dans les traitements informatiques propres aux sciences humaines, les programmes utilisent directement les statistiques officielles. L'analyste du paysage est dans l'heureuse obligation d'élaborer lui-même ses descripteurs. Il s'agit d'une étape fondamentale, dont tout le reste dépend, et qui a longuement retenu l'attention des deux équipes précitées. Revenons au cas de Broze. Les auteurs, procédant par classement et élimination des variables, ont été conduits par les mathématiques à ne retenir dans leur fichier que des descripteurs déterminés directement ou indirectement par la géomorphologie (pente, exposition, altitude relative, altitude absolue, matériel parental, microrelief, etc.), alors que leur formation (agronomie, botanique) les inclinait plutôt à utiliser des critères phytosociologiques et bioclimatiques.

Le traitement des fréquences et la modélisation des paysages. Si le traitement des données est long et pesant, le programme reste, et il est perfectible. On peut l'appliquer à d'autres paysages. L'avantage est double: d'abord, un gain de temps pour les études à venir; ensuite, et c'est cela que nous retiendrons surtout ici, on passe de l'étude monographique, que l'analyse qualitative a du 
mal à dépasser, à des modèles de paysage permettant seuls la classification, la généralisation, la comparaison.

L'analyse numérique appliquée aux paysages en est encore aux prémices. Mais elle donne déjà beaucoup, même à ceux qui ne la pratiquent pas encore. Toutefois, l'analyse multivariée ou à composantes multiples ne peut pas être considérée comme une méthode particulière à l'étude des paysages. En fait, il faut la considérer comme «une technique utilisable pour traiter les grands ensembles de nombres. Elle est donc susceptible de plusieurs types d'application dans le domaine des études du paysage» (G. Allaire). Elle a, semble-t-il, moins d'avenir quand elle est utilisée directement que lorsqu'elle intervient comme support logistique, par exemple dans l'analyse des bilans énergétiques de paysage.

Dans l'instant, les méthodes quantitatives sont autant un outil de réflexion que de traitement des données. Enfin, elles ont l'avantage d'attirer l'attention sur deux «déviations» possibles au sein de la recherche géographique.
La première est de ne considérer l'apport des mathématiques qu'au seul niveau technique, c'est-à-dire comme un simple moyen de traitement statistique de données. Les études de géographie humaine n'ont pas toujours su éviter ce travers. Or, si on domine une méthode, on subit une technique. Les exemples abondent dans la littérature géographique récente.

La seconde est de penser que l'analyse quantitative doit remplacer l'analyse qualitative. La méthode quantitative menace parfois de se refermer sur elle-même, et ses pratiquants ont quelquefois la tentation de l'ésotérisme. Le qualitatif et le quantitatif, dans la mesure où cette distinction a un sens, doivent être dialectiquement liés au sein d'une même démarche scientifique.

S'il y a une «nouvelle géographie», elle ne sera pas une «géographie quantitative», mais elle devra utiliser au maximum l'outil mathématique. Entre ces deux options, se joue l'avenir d'une méthode.

Georges BERTRAND

\section{POSITIONS DE RECHERCHE}

Soucieux de suivre de près l'actualité scientifique, de permettre aux nouvelles recherches de prendre date rapidement et de favoriser l'expression des jeunes chercheurs, l'Espace géographique ouvre une rubrique "Positions de recherche", destinée à accueillir de brefs exposés.

Cette rubrique accueillera d'une part des informations sur les découvertes, les interprétations, les approches ou les techniques nouvelles dont nos lecteurs et nos correspondants auront connaissance.

D'autre part, elle publiera des notes de chercheurs sur leurs propres résultats.

Les notes ne devront pas excéder 2 ou 3 pages dactylographiées; elles ne comporteront d'illustration que si la nouveauté porte sur celle-ci; la date de réception du manuscrit sera publiée.

Cette rubrique n'envisage aucune restriction en fonction des thèmes abordés, ni de la spécialisation des auteurs, du moment que l'apport scientifique permet une meilleure connaissance de l'espace géographique. Comme pour les articles, l'acceptation du manuscrit est du ressort du Comité directeur. 